\title{
Optimal MRI time determination for intracerebral metastases and primary brain tumors after injecting a standard gadobutrol dose: Evidence from a dynamic contrast-enhanced imaging study
}

\section{Jeemin Seo}

Konkuk University Seoul Hospital: Konkuk University Medical Center

Changmok Lim

Konkuk University Seoul Hospital: Konkuk University Medical Center

Kye Young Lee

Konkuk University Seoul Hospital: Konkuk University Medical Center

\section{Young-Cho Koh}

Konkuk University Seoul Hospital: Konkuk University Medical Center

Won-Jin Moon ( $\sim$ mdmoonwj@naver.com )

Konkuk University Medical Center, Konkuk University School of Medicine https://orcid.org/0000-00028925-7376

\section{Research Article}

Keywords: Intracerebral metastases, primary brain tumor, gadobutrol, MRI, Contrast agent

Posted Date: February 23rd, 2022

DOl: https://doi.org/10.21203/rs.3.rs-999065/v1

License: (c) (i) This work is licensed under a Creative Commons Attribution 4.0 International License.

Read Full License 


\section{Abstract}

Background: Recent advances in rapid imaging techniques necessitate the reconsideration of the optimal imaging delay time for contrast-enhanced T1-weighted imaging. The aim of our study was to determine the optimal contrast-enhanced T1-weighted imaging delay time from the obtained time-signal intensity curve (TIC) using gadobutrol in patients with brain metastases, primary brain tumors, and meningiomas.

Methods: This prospective study enrolled 78 patients with brain metastases $(n=39)$, primary brain tumors $(n=22)$, or meningiomas $(n=17)$ who underwent 7-minute dynamic contrast-enhanced imaging with single-dose gadobutrol. Based on the time-to-peak (TTP) derived from the TIC, we selected four different time points for analysis. Lesion conspicuity, enhanced rate (ER) and contrast rate (CR) of 116 index lesions were evaluated. Statistical comparisons were made for the four different time points using the Friedman test.

Results: Maximum TTP (305.20 $\pm 63.47 \mathrm{~s})$ was similar across all three groups $(p=0.342)$. Lesion conspicuity, CR and ER increased over time in all index lesions; however, no significant difference between the 5- and 7-min images was observed. The longest diameter in all groups differed significantly among time points $(p<0.001)$; the perpendicular diameter did not differ between the 5 - and 7 -min images.

Conclusions: Maximum contrast enhancement and lesion conspicuity was achieved 5-7 min after a single gadobutrol injection for brain metastases detection and for primary brain tumor/meningioma evaluation. Acquiring images 5 min after gadobutrol injection is the optimal timing for brain tumor detection during MRI work-up.

\section{Background}

Gadolinium-contrast enhancement magnetic resonance imaging (MRI) is an essential procedure for the diagnosis and progression determination of central nervous system (CNS) tumors [1-3]. It is still often challenging in clinical practice to distinguish single metastasis from a primary brain tumor, and occasionally meningioma $[4,5]$.

While various perfusion imaging techniques have being used to differentiate between brain tumor types, the contrast-enhanced T1WI (CE-T1WI) is considered the standard imaging technique [6]. Recent advances in rapid imaging techniques such as parallel imaging and compressed sensing enable us to obtain MRI sequences with a shorter acquisition time than ever [7]. Thus, the overall imaging time is shortening, forcing us to reconsider the protocol structure in a completely new way. If possible, the interval between contrast agent injection and the CE-T1WI could now be used to perform one or two short but necessary sequences. We need to know the optimal imaging waiting time for CE-T1WI, to utilize this interval better, so a contrast-enhanced fluid-attenuated inversion recovery (FLAIR) or other sequence could be acquired as a supplementary information for tumor characterization. 
The conventional wisdom from a limited number of previous studies [8,9] was that a small enhancing nodule or metastasis could be diagnosed better by delayed imaging and/or using a larger contrast agent dose. However, a retrospective observational study demonstrated that the imaging delay times at three different time points did not affect brain metastases lesion conspicuity following a single gadobutrol dose in a linear function [10]. After a single gadobutrol injection, brain metastases could be detected in both the 1- and 5-min delayed images. In contrast, the 10-min images were ineffective for detection.

A few studies using dynamic contrast-enhanced (DCE) T1-weighted perfusion imaging have vaguely suggested that the time-SI curve shape of primary brain tumors is that of enhancement with a plateau or enhancement with a later washout, not that of steady enhancement $[10,11]$. However, there has been no DCE T1WI study to provide valid information regarding the optimal imaging delay time after GDCA injection. In the era of artificial intelligence-assisted radiology, it would be of importance to know the best available optimal delay time after GDCA injection in order to provide the reliable reference standard.

Therefore, we sought to characterize the time-SI curve of DCE-T1WI using gadobutrol. We also aimed to determine the optimal CE-T1WI delay time from the obtained time-SI curves in patients with brain metastases, primary brain tumors, and meningiomas. We further evaluated the DCE information qualitatively and quantitatively to determine the optimal CE-T1WI delay by extracting data at four time points $(1,3,5$, and 7 min after contrast agent injection).

\section{Methods}

\section{Standards protocol approvals, registrations, and patient consents}

This prospective study was approved by our institutional review board (IRB no. ). Written informed consent was obtained from all participants.

\section{Patients}

We recruited 120 consecutive participants with known or highly suspected brain metastases or highgrade brain tumors previously detected by computed tomography (CT) or MRI between February 2016 and June 2019. The exclusion criteria were as follows: 1) patients with a contraindication to undergo $\mathrm{MRl}$; 2) patients too unstable to undergo MRl; 3) patients with a history of severe allergic or anaphylactoid reaction to contrast agents; 4) patients administered any contrast agent within 24 hours before gadobutrol administration; 5) patients treated with high-dose radiation therapy any time before entering the study; 6) patients with severe cardiovascular disease; 7) pregnant or nursing female patients; 8) patients with severe renal dysfunction (glomerular filtration rate [GFR] $30 \mathrm{~mL} / \mathrm{min}$ ).

Of the 120 participants, we excluded those with no evidence of enhanced lesion $(n=26)$, lesion with unknown etiology ( $n=3)$, and non-tumorous lesions $(n=13)$. Finally, 78 participants were considered (39 with brain metastases, 22 with primary brain tumors (grades III and IV), and 17 with meningioma) (Fig. 1). 


\section{MRI acquisition}

Participants were scanned by the same 3T MRI scanner (Skyra, Siemens Healthineers, Erlangen, Germany) with a 20-channel head coil. The MRI protocol included the following sequences: 1) axial 3D magnetization prepared rapid gradient echo (MPRAGE) (repetition time/echo time [TR/TE] $=1,950 / 3.06$ $\mathrm{ms}, 0.9 \times 0.9 \times 0.5 \mathrm{~mm}) ; 2$ ) axial 2D FLAIR (TR/TE=9,000/95 ms, $0.8 \times 0.8 \times 4.0 \mathrm{~mm}) ; 3)$ axial 2D diffusionweighted imaging (DWl; $b=1,000, T R / T E=8,000 / 68 \mathrm{~ms}$ ); 4) Axial 2D T2WI (TR/TE=3,600/103 ms, $0.8 \times 0.8 \times 4.0 \mathrm{~mm}$ ). In addition, Axial 2D FLAIR and 3D MPRAGE contrast enhanced sequences were obtained after the DCE MRI.

\section{Dynamic contrast-enhanced MRI}

For DCE imaging, axial 3D DCE sequence was acquired with a dynamic series of 65 individual scans with the following parameters: $T R=2.80 \mathrm{~ms}, T E=0.90 \mathrm{~ms}$, flip angle $=10^{\circ}$, average $=1$, field-of-view $=220 \mathrm{~mm}$, slice thickness $=4 \mathrm{~mm}$, matrix $=160 \times 148$, voxel size $=0.75 \times 0.75 \times 4.00 \mathrm{~mm}$, acquisition time $=7 \mathrm{~min}$, and time resolution $=6.5 \mathrm{sec}$. Pre-contrast T1-weighted gradient-echo series (TR=2.97 ms, TE=0.96 ms) with six different flip angles $\left(2-12^{\circ}\right)$ were acquired to generate T1 mapping. A standard gadobutrol dose $(0.1$ $\mathrm{mol} / \mathrm{kg}$ body weight; Bayer Healthcare), followed by a $30-\mathrm{mL}$ saline flush, was automatically injected at a flow rate of $2 \mathrm{~mL} / \mathrm{s}$ with an injector. Injections started after the fourth dynamic scan.

\section{Dynamic contrast-enhanced MRI analysis}

Postprocessing and region of interest (ROI) selection in the DCE imaging data were performed by the NordiclCE software (Version 4.1.3) with 3D T1-volume imaging used for structural imaging. We calculated time-to-peak (TTP) by non-model-based DCE approach. We considered the ROIs mean or maximum TTP to be the estimate of the optimal imaging delay time for lesion detection.

\section{Region of interest analysis for determining the lesions time- to-peak}

To test our hypothesis, index lesions to be investigated in this study were first selected by CE-3D T1 MPRAGE sequence through a consensus review between two radiologists blinded to the patients' clinical information. The size criterion for the ROI analysis was $5 \mathrm{~mm}$ or larger. When we found more than five lesions of the size criterion within the same patient, only five lesions were chosen for the ROI analysis. Accordingly, 74 metastatic nodules, 25 primary brain tumors, and 17 meningiomas were selected as index lesions for the ROI analysis (totaling 116 lesions). Each ROI was placed on an enhanced lesion on the CE3D T1 MPRAGE image and then transferred to the co-registered DCE parametric map to calculate the DCE parameters mentioned above.

\section{Qualitative image quality assessment for the index lesion at different time points}

The image slices for index lesions were collected from the DCE imaging data at four time points after contrast agent injection. The acquired images were displayed simultaneously in a random order for the 
qualitative assessment.

One neuroradiologist (22 years of neuroradiology experience) and one radiology trainee (3 years of radiology experience) - and both blinded to the DCE sequence image time delay and the patient clinical information-independently performed a side-by-side comparison of the four images of each index lesion to determine lesion conspicuity. The lesion conspicuity was evaluated for each image and rated on a four-point scale: 0 , lesion not visualized; 1 , poor visualization; 2 , moderate visualization; 3 , good clear visualization.

\section{Quantitative assessment of image quality for the index lesion according to the different time points}

As an image rating quality assessment, one of the raters performed a second quantitative ROI analysis, evaluating the DCE imaging data index lesions at the four time points two weeks after the initial qualitative assessment. The ROI data was used to calculate the contrast rate (CR) and enhancement rate (ER) on images acquired 1, 3, 5, and 7 min after contrast agent injection [12]. The CR and ER of the lesion were defined as follows [12]:

$\underline{\mathrm{CR}}(\underline{\%}) \equiv($ post-contrast SI_lesion - post-contrast SI_white matter) $\times 100 /$ post-contrast white matter

ER $(\%) \equiv($ post-contrast SI_lesion - baseline gray matter) $\times 100$ / baseline gray matter

The lesion and white matter ROIs were measured on all images, selecting the most enhanced solid portion. We strictly avoided including vessels, other strongly contrasting structures, or artifacts in the ROI. The ROI was positioned over the entire lesion enhancing area in homogeneous lesions or over the maximal enhancing area in inhomogeneous lesions. For extensive lesions with an irregular boundary, the $\mathrm{ROI}$ was positioned to include the largest possible proportion of the lesion at the given slice. We used the image obtained immediately after contrast injection $(0 \mathrm{~min})$ from the DCE data for baseline gray matter signal intensity. The ROI on the image acquired one minute after contrast agent injection was copied to the 3,5 , and 7 min images.

Additionally, we measured the longest diameter and perpendicular diameter of the index lesions on all selected images. We chose to measure the two-dimensional diameter for the tumor size assessment because current practice such as response criteria for brain metastasis from the RANO group is still based on an unidimensional measurement [13]. Eleven lesions (seven metastases, three primary brain tumors, one meningioma) were excluded because they could not be clearly identified on images acquired 1 min after contrast injection. Thus, diameter measurements were performed on 105 index lesions.

\section{Statistical analysis}

Statistical analysis was performed using the IBM SPSS Statistics for Windows, Version 24.0 (IBM Corp., Armonk, NY, USA) and MedCalc (Versions 19.1). Differences with $p<0.05$ were considered statistically significant. 
The Friedman test was used to evaluate the differences in index lesion conspicuity, CR, ER, diameter measurements among the four time points $(1,3,5$, and 7 min after contrast agent injection). We used the Wilcoxon rank-sum test for post hoc multiple pair-wise comparisons correction, with $p$-value $<0.008$ as a Bonferroni-corrected $\mathrm{p}$ value.

Agreement between the two raters for lesion conspicuity was calculated using the $\mathrm{k}$ statistics, with $\mathrm{k}<0.2$, 0.2 to $0.4,>0.4$ to $0.6,>0.6$ to 0.8 , and $>0.8$ to 1.0 , representing poor, fair, moderate, good, and excellent agreement, respectively.

\section{Result}

\section{Determination of the peak time from the dynamic contrast- enhanced data}

In brain metastases and primary brain tumors, mean TTP tended to be longer than in meningioma (185.3 $\pm 84.4 \mathrm{~s}$ and $204.32 \pm 118.32 \mathrm{~s}$ vs. $138.61 \pm 106.08 \mathrm{~s}$, respectively, $p=0.089)$. However, maximum TTP was similar in all three groups (305.20 $\pm 63.47 \mathrm{~s} ; p=0.342)$.

\section{Qualitative assessment of tumor contrast enhancement at the four time points}

We selected four time points to extract images from DCE data according to the TTP analysis described above. These were $1 \mathrm{~min}(60 \mathrm{sec}), 3 \mathrm{~min}$ (180 sec), $5 \mathrm{~min}(300 \mathrm{sec})$, and $7 \mathrm{~min}(420 \mathrm{sec})$ after contrast agent injection.

The grade of the lesion conspicuity (degree of contrast enhancement) at the four time points after contrast agent injection showed that the lesion conspicuity increased with time in all index lesions $(p<0.001)$. Post hoc comparisons for all groups combined and for the metastasis group showed that the lesion conspicuity grades in the 5-min and 7-min images were similar.

Primary brain tumor and meningioma on images acquired $1 \mathrm{~min}$ after contrast agent injection had significantly lower lesion conspicuity grades than those on images acquired after 3,5, and 7 min. However, the images acquired after 3, 5, and 7 min were similar.

Inter-rater lesion conspicuity agreement was 0.46 (95\% confidence interval [Cl], 0.28-0.64), 0.46 (0.27$0.64), 0.64$ (0.46-0.83), $0.65(0.48-0.81)$ for images acquired after $1,3,5$, and $7 \mathrm{~min}$, respectively. The inter-rater agreement of lesion conspicuity for images acquired after 5 and 7 min was better than for images acquired after 1 and 3 min.

\section{Quantitative assessment of the tumor contrast enhancement at the four time points}


CR and ER differed significantly between primary brain tumors and metastases images acquired 1, 3, and 5 min after contrast agent injection. However, we found no noticeable difference in CR or ER between images acquired after 5 and 7 min (Fig. 2 and Fig. 3)(Supplementary Table 1).

The longest diameter in all groups differed significantly between time points (Friedman test, $p<0.001$ for brain metastases and primary brain tumor, $\mathrm{p}<0.019$ for meningioma), except for primary brain tumors on images acquired after 3 and 5 min in pair-wise comparison ( $p=0.102)$ (Fig. 4a). The perpendicular-longest diameter increased in size on metastases images acquired up to $5 \mathrm{~min}$ after contrast agent injection but was similar on images acquired after 5 and 7 min (Fig. 4b). Post-hoc comparison revealed that primary brain tumors showed no significant difference between the perpendicular-longest diameter on images acquired between $3 \mathrm{~min}, 5 \mathrm{~min}$ and $7 \mathrm{~min}$ time points. The perpendicular-longest diameter of meningioma was not different across the images acquired at all time points (Fig. 4b).

\section{Discussion}

In this study, we found that the maximum TTP of brain metastases, primary brain tumors, and meningiomas was approximately 5 min. Qualitative and quantitative assessments of contrast enhancement supported this finding, showing that images acquired 5 and 7 min after contrast agent injection were comparable in terms of lesion conspicuity and enhancement (Fig. 5). Enhancement on images acquired after 7 min was only marginally better for the longest diameter measurement of the lesions.

Our observation of the gradual contrast enhancement in the primary brain tumor and brain metastases until 5 min after contrast agent injection when using DCE imaging partly contradicts a previous study that reported images acquired after 1 min showed an equal contrast effect to the images acquired after 5 $\min [10]$. The researchers also found that images after 1- and 5-min delays showed a higher contrast effect than images acquired after a 10-min delay [10]. Despite our result rejecting the quality of images after $1 \mathrm{~min}$, the gradual enhancement of the lesions until $5 \mathrm{~min}$ after contrast injection, and the comparability between images acquired after 5 and $7 \mathrm{~min}$, supports the previous study's assumption that waiting for five minutes after contrast agent injection was effective for intracerebral metastases detection [10]

Intracranial brain tumors and metastases reportedly revealed persistent or slow gradual enhancement over time [14]. However, recent DCE studies showed that primary brain tumors might exhibit early enhancement and delayed washout, not a plateau $[11,15]$. Engelhorn et al. studied DCE features of experimental gliomas and found that waiting eight minutes after contrast agent injection revealed $84 \%$ of the tumors and seemed to be a practical clinical compromise between imaging delay time and detection rate [16]. A few DCE imaging studies have dealt with brain metastases [17-19], but none has determined the peak enhancement time. Our study was the first to show that the optimal brain metastases enhancement could be achieved 5 min after contrast injection based on DCE imaging data. 
Differences in enhancement characteristics between the three groups might be attributable to the degree of hypervascularity, presence of dural arterial supply, difference in histologic type, and abundance of glycosaminoglycan [20]. The maximum TTP in our study was much longer than the mean TTP of the target lesion, understandable considering that the tumor enhancement curve shows a different pattern depending on the tumor portion [11]. Given tumor heterogeneity, maximum TTP might represent the entire tumor more comprehensively than mean TTP does.

The imaging assessment also confirmed that images acquired after 5 and 7 min were comparable in lesion conspicuity, ER, and CR. Our study is also in line with a previous study [10] that found lesion conspicuity and the quantitative results to become prominent over the first $5 \mathrm{~min}$ after contrast injection but not extending to the point of 7 min after contrast injection. However, Yuh et al. reported that small brain metastases were better detected on images acquired after a far longer waiting time $(10,20$, and 30 min) [8]. Cohen-Inbar et al. also discovered more lesions $20 \mathrm{~min}$ after contrast agent injection, especially in association with the posterior circulation [21]. The mean diameter of the observed lesions was approximately $3 \mathrm{~mm}$. Accordingly, a recent study recommended waiting 10-15 min before acquiring images to detect brain metastases [22].

However, higher sensitivity for brain metastases by any method could be at the risk of a higher falsepositive rate, as when using a higher GDCA dose [23]. Besides brain metastases, solid enhancing nodules can be observed in various diseases, including vasculitis, demyelinating plaques, and infections [24]. Non-tumorous lesions with blood-brain barrier leakage can particularly benefit from delayed images, unlike brain metastases or primary brain tumors that inherently have pathological hypervascularity[25].

Our diameter measurement also revealed that waiting 5 or 7 min did not affect the measurement power of the tumor dimensions. Brain metastases response assessment is important but was not standardized until the response assessment in neuro-oncology brain metastases (RANO-BM) criteria were recently introduced [26]. Measurable disease is defined in RANO-BM as a contrast-enhancing lesion that can be measured in at least two plane dimensions, with a minimum size of $10 \mathrm{~mm}$. At least a $30 \%$ decrease in the sum longest diameter of a CNS target lesion was defined as a partial response while a $20 \%$ or more increase in the sum longest diameter of a CNS target lesion was defined as progressive disease [26]. Thus, it is of paramount importance to measure accurately the tumor largest and perpendicular diameters in contrast-enhanced images. Our findings suggest that the appropriate cutoff for the waiting time from injection would be five minutes.

Our results have a potential implication in the era of Al-assisted radiology. The observations in our study could be used as a reference to develop Al-assisted diagnostic tool that deals with heterogeneously obtained imaging data. Second, our result can be used to optimize MRI protocol for quality of patient care and for throughput in MRI. In research hospitals, various sequences could be inserted between contrast agent injection and the contrast-enhanced image acquisition $5 \mathrm{~min}$ later. These could include dynamic susceptibility contrast (DSC), DCE, contrast-enhanced FLAIR, and more. In more rural settings, a standard enhanced FLAIR for leptomeningeal pathology could be inserted during the 5 min waiting time. Above all, 
accurate knowledge of the optimal waiting time could help to improve the patient's comfort level by reducing the MRI examination time.

Our study has some limitations. First, although we evaluated the entire DCE time-intensity curve for seven minutes, one could argue that estimating the time-intensity curve is inadequate. However, given the tumor pathological innate vascularity, our findings could reasonably represent the nature of the tumorous conditions studied. Second, several factors might affect the contrast enhancement, including MRI sequence parameters, post-processing software programs, and contrast agents. We acknowledge that different contrast agents, such as the macrocyclic ionic agent, might show different results. Nevertheless, given the binding nature of the macrocyclic ionic agent to glycosaminoglycan within the tumor, we assume that such a GDCA might accentuate our results rather than disapprove them [27]. This recent paper found no difference in DCE parameters between gadobutrol and gadoterate dimeglumin [27].

\section{Conclusion}

In conclusion, maximum values of TTP, CR, ER, and lesion conspicuity can be achieved 5-7 min after a single-dose gadobutrol injection for brain metastases detection and for primary brain tumor/meningioma. Our findings suggest that, to optimize brain metastasis and primary brain tumor/meningioma detection on MRI workup, images should be acquired 5 min after contrast agent injection.

\section{Declarations}

\section{Ethics approval and consent to participate}

- This prospective study was approved by institutional review board of Konkuk university medical center (IRB no. 1140098)

- Written informed consent was obtained from all participants

\section{Consent to publication}

- Written informed consent was obtained from all participants

\section{Availability of data and materials}

- The data that support the findings of this study are available on request from the corresponding author, Moon WJ. The data are not publicly available due to their containing information that could compromise the privacy of research participants 


\section{Competing interests}

- The authors declare that they have no competing interests.

\section{Funding}

- This study was supported by a research grant of Bayer.

- (funding was mainly used for interpretation of data)

\section{Author contributions}

- WJ conceptualized the study, perform major contribution in writing the manuscript, and supervised analysis.

- YC, KY conceptualized the study and collected/provided patient data.

- JM analyzed the patient data and performed major contribution in writing the manuscript.

- CM analyzed the patient data and performed analysis using software.

- All authors read and approved the final manuscript

\section{Acknowledgements}

- This work was supported by the Bayer Healthcare.

\section{References}

1. J.W. Choi, W.-J. Moon, Gadolinium Deposition in the Brain: Current Updates, Korean Journal of Radiology 20(1) (2019).

2. E.S. Kim, J.H. Chang, H.S. Choi, J. Kim, S.K. Lee, Diagnostic Yield of Double-Dose Gadobutrol in the Detection of Brain Metastasis: Intraindividual Comparison with Double-Dose Gadopentetate Dimeglumine, American Journal of Neuroradiology 31(6) (2010) 1055-1058.

3. U.I. Attenberger, V.M. Runge, C.B. Jackson, S. Baumann, K. Birkemeier, H.J. Michaely, S.O. Schoenberg, M.F. Reiser, B.J. Wintersperger, Comparative evaluation of lesion enhancement using 1 $M$ gadobutrol vs. 2 conventional gadolinium chelates, all at a dose of $0.1 \mathrm{mmol} / \mathrm{kg}$, in a rat brain tumor model at 3 T, Invest Radiol 44(5) (2009) 251-6. 
4. M. Artzi, I. Bressler, D. Ben Bashat, Differentiation between glioblastoma, brain metastasis and subtypes using radiomics analysis, J Magn Reson Imaging 50(2) (2019) 519-528.

5. M.D. Lee, G.L. Baird, L.C. Bell, C.C. Quarles, J.L. Boxerman, Utility of Percentage Signal Recovery and Baseline Signal in DSC-MRI Optimized for Relative CBV Measurement for Differentiating Glioblastoma, Lymphoma, Metastasis, and Meningioma, AJNR Am J Neuroradiol 40(9) (2019) 1445-1450.

6. J.E. Park, H.S. Kim, Current Applications and Future Perspectives of Brain Tumor Imaging, Journal of the Korean Society of Radiology 81(3) 467-487.

7. E. Sartoretti, T. Sartoretti, C. Binkert, A. Najafi, A. Schwenk, M. Hinnen, L. van Smoorenburg, B. Eichenberger, S. Sartoretti-Schefer, Reduction of procedure times in routine clinical practice with Compressed SENSE magnetic resonance imaging technique, PLoS One 14(4) (2019) e0214887.

8. W.T. Yuh, E.T. Tali, H.D. Nguyen, T.M. Simonson, N.A. Mayr, D.J. Fisher, The effect of contrast dose, imaging time, and lesion size in the MR detection of intracerebral metastasis, AJNR Am J Neuroradiol 16(2) (1995) 373-80.

9. N. Anzalone, S. Gerevini, R. Scotti, P. Vezzulli, P. Picozzi, Detection of cerebral metastases on magnetic resonance imaging: intraindividual comparison of gadobutrol with gadopentetate dimeglumine, Acta Radiologica 50(8) (2009) 933-940.

10. J. Jeon, J. Choi, H. Roh, W.-J. Moon, Effect of imaging time in the magnetic resonance detection of intracerebral metastases using single dose gadobutrol, Korean Journal of Radiology 15(1) (2014) 145-150.

11. C.H. Suh, H.S. Kim, Y.J. Choi, N. Kim, S.J. Kim, Prediction of Pseudoprogression in Patients with Glioblastomas Using the Initial and Final Area Under the Curves Ratio Derived from Dynamic Contrast-Enhanced T1-Weighted Perfusion MR Imaging, American Journal of Neuroradiology 34(12) (2013) 2278-2286.

12. K. Furutani, M. Harada, M. Mawlan, H. Nishitani, Difference in enhancement between spin echo and 3-dimensional fast spoiled gradient recalled acquisition in steady state magnetic resonance imaging of brain metastasis at 3-T magnetic resonance imaging, Journal of computer assisted tomography 32(2) (2008) 313-319.

13. N.U. Lin, E.Q. Lee, H. Aoyama, I.J. Barani, D.P. Barboriak, B.G. Baumert, M. Bendszus, P.D. Brown, D.R. Camidge, S.M. Chang, Response assessment criteria for brain metastases: proposal from the RANO group, The lancet oncology 16(6) (2015) e270-e278.

14. K.R. Maravilla, J.A. Maldjian, I.M. Schmalfuss, M.J. Kuhn, B.C. Bowen, F.J. Wippold, 2nd, V.M. Runge, M.V. Knopp, S. Kremer, L.J. Wolansky, N. Anzalone, M. Essig, L. Gustafsson, Contrast enhancement of central nervous system lesions: multicenter intraindividual crossover comparative study of two MR contrast agents, Radiology 240(2) (2006) 389-400.

15. B.C. Jung, J. Arevalo-Perez, J.K. Lyo, A.I. Holodny, S. Karimi, R.J. Young, K.K. Peck, Comparison of Glioblastomas and Brain Metastases using Dynamic Contrast-Enhanced Perfusion MRI, J Neuroimaging 26(2) (2016) 240-6. 
16. T. Engelhorn, M.A. Schwarz, I.Y. Eyupoglu, S.P. Kloska, T. Struffert, A. Doerfler, Dynamic Contrast Enhancement of Experimental Glioma: An Intra-individual Comparative Study to Assess the Optimal Time Delay, Academic Radiology 17(2) (2010) 188-193.

17. G. Kuchcinski, E. Le Rhun, A.B. Cortot, E. Drumez, R. Duhal, M. Lalisse, J. Dumont, R. Lopes, J.P. Pruvo, X. Leclerc, C. Delmaire, Dynamic contrast-enhanced MR imaging pharmacokinetic parameters as predictors of treatment response of brain metastases in patients with lung cancer, European Radiology 27(9) (2017) 3733-3743.

18. Y.B. Xi, X.W. Kang, N. Wang, T.T. Liu, Y.Q. Zhu, G. Cheng, K. Wang, C. Li, F. Guo, H. Yin, Differentiation of primary central nervous system lymphoma from high-grade glioma and brain metastasis using arterial spin labeling and dynamic contrast-enhanced magnetic resonance imaging, European Journal of Radiology 112(European journal of radiology 835 2014) (2019) 59-64.

19. J.I. Traylor, D.C.A. Bastos, D. Fuentes, M. Muir, R. Patel, V.A. Kumar, R.J. Stafford, G. Rao, S.S. Prahhu, Dynamic Contrast-Enhanced MRI in Patients with Brain Metastases Undergoing Laser Interstitial Thermal Therapy: A Pilot Study, American Journal of Neuroradiology 40(9) (2019) 1451-1457.

20. A. Engelhardt, Detection of acid mucopolysaccharides in human brain tumors by histochemical methods, Acta Neuropathologica 49(3) (1980) 199-203.

21. O. Cohen-Inbar, Z. Xu, B. Dodson, T. Rizvi, C.R. Durst, S. Mukherjee, J.P. Sheehan, Time-delayed contrast-enhanced MRI improves detection of brain metastases: a prospective validation of diagnostic yield, Journal of neuro-oncology 130(3) (2016) 485-494.

22. M. Kushnirsky, V. Nguyen, J.S. Katz, J. Steinklein, L. Rosen, C. Warshall, M. Schulder, J.P. Knisely, Time-delayed contrast-enhanced MRI improves detection of brain metastases and apparent treatment volumes, J Neurosurg 124(2) (2016) 489-95.

23. O. Togao, A. Hiwatashi, K. Yamashita, K. Kikuchi, T. Yoshiura, H. Honda, Additional MR contrast dosage for radiologists' diagnostic performance in detecting brain metastases: a systematic observer study at 3 T, Jpn J Radiol 32(9) (2014) 537-44.

24. R.J. Packer, D. Schiff, Neuro-oncology, John Wiley \& Sons2012.

25. G.A. Rosenberg, Neurological diseases in relation to the blood-brain barrier, J Cereb Blood Flow Metab 32(7) (2012) 1139-51.

26. N.U. Lin, E.Q. Lee, H. Aoyama, I.J. Barani, D.P. Barboriak, B.G. Baumert, M. Bendszus, P.D. Brown, D.R. Camidge, S.M. Chang, J. Dancey, E.G.E. de Vries, L.E. Gaspar, G.J. Harris, F.S. Hodi, S.N. Kalkanis, M.E. Linskey, D.R. Macdonald, K. Margolin, M.P. Mehta, D. Schiff, R. Soffietti, J.H. Suh, M.J. van den Bent, M.A. Vogelbaum, P.Y. Wen, R.A. Neuro-Oncology, Response assessment criteria for brain metastases: proposal from the RANO group, Lancet Oncol 16(6) (2015) E270-E278.

27. J.E. Park, J.Y. Kim, H.S. Kim, W.H. Shim, Comparison of Dynamic Contrast-Enhancement Parameters between Gadobutrol and Gadoterate Meglumine in Posttreatment Glioma: A Prospective Intraindividual Study, American Journal of Neuroradiology 41(11) (2020) 2041-2048.

\section{Figures}




\section{Figure 1}

Flowchart of the study population.

\section{Figure 2}

Differences in contrast rate between time points after contrast agent injection based on tumor type (ns: not significant, $*<0.008, * \star<0.001, * \star \star<0.0001)$ Statistically significant $p$ is 0.008 as a Bonferroni-corrected $p$ value.

\section{Figure 3}

Differences in enhancement rate between time points after contrast agent injection based on tumor type. (ns: not significant, $*<0.008, * *<0.001, * * *<0.0001$ )
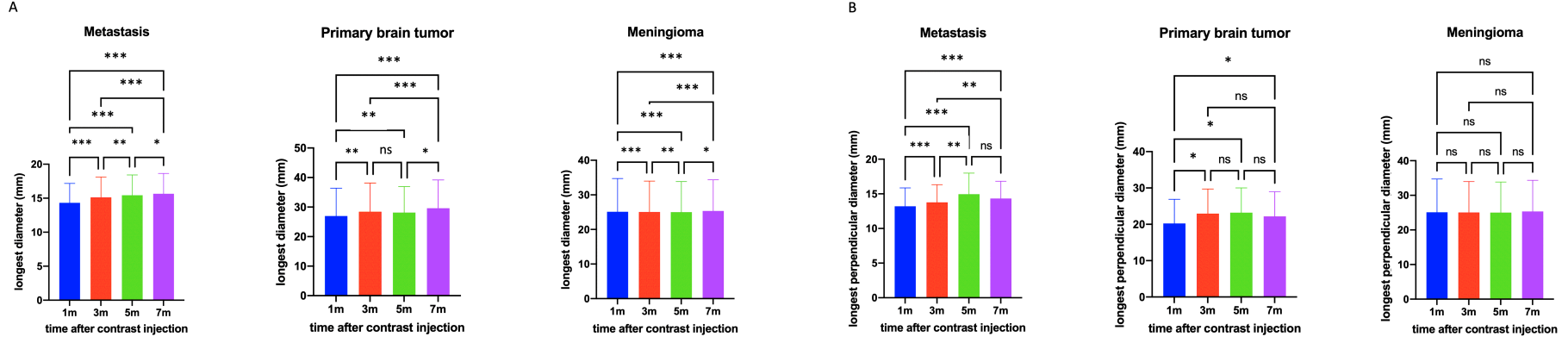

Figure 4

Differences between time points in the longest diameter (4a) and the longest perpendicular diameter (4b). (ns: not significant, $*<0.008, * *<0.001, * * *<0.0001$ )

\section{Figure 5}

(a) A 69-year-old man displaying a brain metastasis from a lung cancer.

3D dynamic contrast enhanced (DCE) axial images acquired 1, 3, 5, and 7 min after injection of a single standard gadobutrol dose. The lesion on images acquired after 5 and 7 min appears similar in terms of 
conspicuity.

(b) A 77-year-old man or woman displaying a primary brain tumor (glioblastoma).

The lesion shows gradual increment of contrast enhancement that reaches the highest conspicuity 7 min after contrast agent injection.

\section{Supplementary Files}

This is a list of supplementary files associated with this preprint. Click to download.

- SupplementaryTable1.docx 